\title{
On oriented graphs with minimal skew energy
}

\author{
Shicai Gong ${ }^{a * \dagger}$, Xueliang $\mathrm{Li}^{b \ddagger}$ and Guanghui $\mathrm{Xu}^{a \S}$ \\ a. Zhejiang A \& F University, Hangzhou, 311300, P. R. China \\ b. Center for Combinatorics and LPMC-TJKLC, Nankai University, \\ Tianjin 300071, P. R. China
}

\begin{abstract}
Let $S\left(G^{\sigma}\right)$ be the skew-adjacency matrix of an oriented graph $G^{\sigma}$. The skew energy of $G^{\sigma}$ is defined as the sum of all singular values of its skewadjacency matrix $S\left(G^{\sigma}\right)$. In this paper, we first deduce an integral formula for the skew energy of an oriented graph. Then we determine all oriented graphs with minimal skew energy among all connected oriented graphs on $n$ vertices with $m(n \leq m<2(n-2))$ arcs, which is an analogy to the conjecture for the energy of undirected graphs proposed by Caporossi et al. [G. Caporossi, D. Cvetković, I. Gutman, P. Hansen, Variable neighborhood search for extremal graphs. 2. Finding graphs with external energy, J. Chem. Inf. Comput. Sci. 39 (1999) 984-996.].
\end{abstract}

Keywords: oriented graph; graph energy; skew energy; skew-adjacency matrix; skew characteristic polynomial.

AMS subject classification 2010: 05C50, 15A18

\section{Introduction}

Let $G^{\sigma}$ be a digraph that arises from a simple undirected graph $G$ with an orientation $\sigma$, which assigns to each edge of $G$ a direction so that $G^{\sigma}$ becomes an oriented graph,

\footnotetext{
${ }^{*}$ Corresponding author. E-mail addresses: scgong@zafu.edu.cn(S. Gong); lxl@nankai.edu.cn(X. Li); ghxu@zafu.edu.cn(G. Xu).

†Supported by Zhejiang Provincial Natural Science Foundation of China(No. Y12A010049).

$\ddagger$ Supported by National Natural Science Foundation of China(No. 10831001).

$\S$ Supported by National Natural Science Foundation of China(No. 11171373).
} 
or a directed graph. Then $G$ is called the underlying graph of $G^{\sigma}$. Let $G^{\sigma}$ be an undirected graph with vertex set $V\left(G^{\sigma}\right)=\left\{v_{1}, v_{2}, \cdots, v_{n}\right\}$. Denote by $(u, v)$ an arc, of $G^{\sigma}$, with tail $u$ and head $v$. The skew-adjacency matrix related to $G^{\sigma}$ is the $n \times n$ matrix $S\left(G^{\sigma}\right)=\left[s_{i j}\right]$, where the $(i, j)$ entry satisfies:

$$
s_{i j}= \begin{cases}1, & \text { if }\left(\mathrm{v}_{\mathrm{i}}, \mathrm{v}_{\mathrm{j}}\right) \in \mathrm{G}^{\sigma} ; \\ -1, & \text { if }\left(\mathrm{v}_{\mathrm{j}}, \mathrm{v}_{\mathrm{i}}\right) \in \mathrm{G}^{\sigma} \\ 0, & \text { otherwise. }\end{cases}
$$

The skew energy of an oriented graph $G^{\sigma}$, introduced by Adiga, Balakrishnan and So in [1] and denoted by $\mathcal{E}_{S}\left(G^{\sigma}\right)$, is defined as the sum of all singular values of $S\left(G^{\sigma}\right)$. Because the skew-adjacency matrix $S\left(G^{\sigma}\right)$ is skew-symmetric, the eigenvalues $\left\{\lambda_{1}, \lambda_{2}, \cdots, \lambda_{n}\right\}$ of $S\left(G^{\sigma}\right)$ are all purely imaginary numbers. Consequently, the skew energy $\mathcal{E}_{S}\left(G^{\sigma}\right)$ is the sum of the absolute values of its eigenvalues, i.e.,

$$
\mathcal{E}_{S}\left(G^{\sigma}\right)=\sum_{i=1}^{n}\left|\lambda_{i}\right|
$$

which has the same expression as that of the energy of an undirected graph with respect to its adjacent matrix; see e.g. [14].

The work on the energy of a graph can be traced back to 1970's [10] when Gutman investigated the energy with respect to the adjacency matrix of an undirected graph, which has a still older chemical origin; see e.g. [6]. Then much attention has been devoted to the energy of the adjacency matrix of a graph; see e.g. [2, 3, 4, 17, 12, 13, 11, 18, 21, 22, 25], and the references cited therein. For undirected graphs, Caporossi, Cvetković, Gutman and Hansen [5] proposed a conjecture for the minimum energy as follows.

Conjecture 1. Let $G$ be the graph with minimum energy among all connected graphs with $n \geq 6$ vertices and $m(n \leq m \leq 2(n-2))$ edges. Then $G$ is $O_{n, m}$ if $m \leq n+\left\lfloor\frac{n-7}{2}\right\rfloor$; and $B_{n, m}$ otherwise, where $O_{n, m}$ and $B_{n, m}$ are respectively the underlying graphs of the oriented graphs $O_{n, m}^{+}$and $B_{n, m}^{+}$given in Fig. 1.1.

This conjecture was proved to be true for $m=n-1,2(n-2)$ by Caporossi et al. ([5], Theorem 1), and $m=n$ by Hou [17]. In [22], Li, Zhang and Wang confirmed this conjecture for bipartite graphs. Conjecture 1 has not yet been solved completely.

Recently, in analogy to the energy of the adjacency matrix, a few other versions of graph energy were introduced in the mathematical literature, such as Laplacian energy [16], signless Laplacian energy [15] and skew energy [1].

In [1], Adiga et.al. obtained the skew energies of directed cycles under different orientations and showed that the skew energy of a directed tree is independent of 
its orientation, which is equal to the energy of its underlying tree. Naturally, the following question is interesting:

Question: Denote by $M$ a class of oriented graphs. Which oriented graphs have the extremely skew energy among all oriented graphs of $M$ ?

Hou et al. [20] determined the oriented unicyclic graphs with the maximal and minimal skew energies. Zhu [26] determined the oriented unicyclic graphs with the first $\left\lfloor\frac{n-9}{2}\right\rfloor$ largest skew energies. Shen el al. [23] determined the bicyclic graphs with the maximal and minimal energies. Gong and $\mathrm{Xu}[9]$ determined the 3-regular graphs with the optimum skew energy, and Tian [24] determined the hypercubes with the optimum skew energy. In the following we will study the minimal skew energy graphs of order $n$ and size $m$.

At first, we need some notations. Denote by $K_{n}, S_{n}$ and $C_{n}$ the complete undirected graph, the undirected star and the undirected cycle on $n$ vertices, respectively. Let $O_{n, m}^{+}$be the oriented graph on $n$ vertices which is obtained from the oriented star $S_{n}^{\sigma}$ by adding $m-n+1$ arcs such that all those arcs have a common vertex; see Fig. 1.1, where $v_{1}$ is the tail of each arc incident to it and $v_{2}$ is the head of each arc incident to it, and $B_{n, m}^{+}$, the oriented graph obtained from $O_{n, m+1}^{+}$by deleting the arc $\left(v_{1}, v_{2}\right)$. Denote by $O_{n, m}$ and $B_{n, m}$ the underlying graphs of $O_{n, m}^{+}$and $B_{n, m}^{+}$, respectively. Notice that both $O_{n, m}^{+}$and $B_{n, m}^{+}$contain $n$ vertices and $m$ arcs.
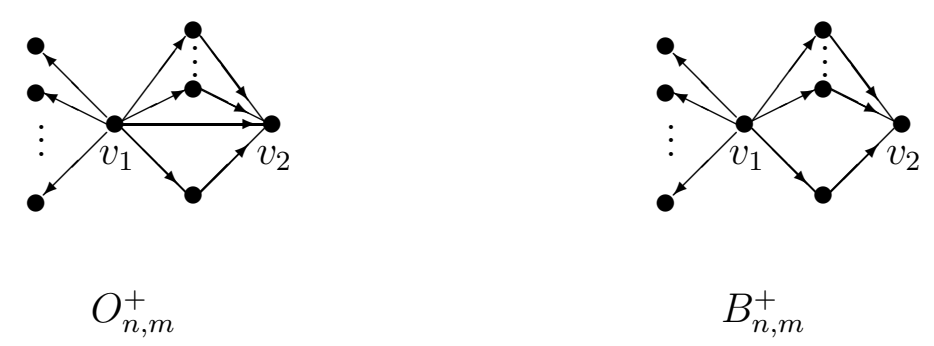

Fig. 1.1. Two oriented graphs $O_{n, m}^{+}$and $B_{n, m}^{+}$.

In this paper, we first deduce an integral formula for the skew energy of an oriented graph. Then we study the question above and determine all oriented graphs with minimal skew energy among all connected oriented digraphs on $n$ vertices with $m(n \leq m<2(n-2))$ arcs. Interestingly, our result is an analogy to Conjecture 1.

Theorem 1 Let $G^{\sigma}$ be an oriented graph with minimal skew energy among all oriented graphs with $n$ vertices and $m(n \leq m<2(n-2))$ arcs. Then, up to isomorphism, $G^{\sigma}$ is 
(1) $O_{n, m}^{+}$if $m<\frac{3 n-5}{2}$;

(2) either $B_{n, m}^{+}$or $O_{n, m}^{+}$if $m=\frac{3 n-5}{2}$; and

(3) $B_{n, m}^{+}$otherwise.

\section{Integral formula for the skew energy}

In this section, based on the formula established by Adiga et al. 11, we deduce an integral formula for the skew energy of an oriented graph, which is an analogy to the Coulson integral formula for the energy of an undirected graph. Firstly, we introduce some notations and preliminary results.

An even cycle $C$ in an oriented graph $G^{\sigma}$ is called oddly oriented if for either choice of direction of traversal around $C$, the number of edges of $C$ directed in the direction of the traversal is odd. Since $C$ is even, this is clearly independent of the initial choice of direction of traversal. Otherwise, such an even cycle $C$ is called as evenly oriented. (Here we do not involve the parity of the cycle with length odd. The reason is that it depends on the initial choice of direction of traversal.)

A "basic oriented graph" is an oriented graph whose components are even cycles and/or complete oriented graphs with exactly two vertices.

Denote by $\phi\left(G^{\sigma} ; x\right)$ the skew characteristic polynomial of an oriented graph $G^{\sigma}$, which is defined as

$$
\phi\left(G^{\sigma} ; x\right)=\operatorname{det}\left(x I_{n}-S\left(G^{\sigma}\right)\right)=\sum_{i=0}^{n} a_{i}\left(G^{\sigma}\right) x^{n-i},
$$

where $I_{n}$ denotes the identity matrix of order $n$. The following result is a cornerstone of our discussion below, which determines all coefficients of the skew characteristic polynomial of an oriented graph in terms of its basic oriented subgraphs; see [19, Theorem 2.4] for an independent version.

Lemma 2 [8, Corollary 2.3] Let $G^{\sigma}$ be an oriented graph on $n$ vertices, and let the skew characteristic polynomial of $G^{\sigma}$ be

$\phi\left(G^{\sigma}, \lambda\right)=\sum_{i=0}^{n}(-1)^{i} a_{i} \lambda^{n-i}=\lambda^{n}-a_{1} \lambda^{n-1}+a_{2} \lambda^{n-2}+\cdots+(-1)^{n-1} a_{n-1} \lambda+(-1)^{n} a_{n}$.

Then $a_{i}=0$ if $i$ is odd; and

$$
a_{i}=\sum_{\mathscr{H}}(-1)^{c^{+}} 2^{c} \text { if } i \text { is even, }
$$


where the summation is over all basic oriented subgraphs $\mathscr{H}$ of $G^{\sigma}$ having $i$ vertices and $c^{+}$and $c$ are respectively the number of evenly oriented even cycles and even cycles contained in $\mathscr{H}$.

Let $G=(V(G), E(G))$ be a graph, directed or not, on $n$ vertices. Then denote by $\Delta(G)$ the maximum degree of $G$ and set $\Delta\left(G^{\sigma}\right)=\Delta(G)$. An $r$-matching in a graph $G$ is a subset of $r$ edges such that every vertex of $V(G)$ is incident with at most one edge in it. Denote by $M(G, r)$ the number of all $r$-matchings in $G$ and set $M(G, 0)=1$.

Denote by $q(G)$ the number of quadrangles in a undirected graph $G$. Then as a consequence of Lemma 2, we have

Theorem 3 Let $G^{\sigma}$ be an oriented graph containing $n$ vertices and $m$ arcs. Suppose

$$
\phi\left(G^{\sigma}, \lambda\right)=\sum_{i=0}^{n}(-1)^{i} a_{i}\left(G^{\sigma}\right) \lambda^{n-i}
$$

Then $a_{0}\left(G^{\sigma}\right)=1, a_{2}\left(G^{\sigma}\right)=m$ and $a_{4}\left(G^{\sigma}\right) \geq M(G, 2)-2 q(G)$ with equality if and only if all oriented quadrangles of $G^{\sigma}$ are evenly oriented.

Proof. The result follows from Lemma 2 and the fact that each arc corresponds a basic oriented graph having 2 vertices, and each basic oriented graph having 4 vertices is either a 2-matching or a quadrangle.

Furthermore, as well-known, the eigenvalues of an arbitrary real skew symmetric matrix are all purely imaginary numbers and occur in conjugate pairs. Henceforth, Lemma 2 can be strengthened as follows, which will provide much convenience for our discussion below.

Lemma 4 Let $G^{\sigma}$ be an oriented graph of order $n$. Then each coefficient of the skew characteristic polynomial

$$
\phi\left(G^{\sigma}, \lambda\right)=\sum_{i=0}^{\left\lfloor\frac{n}{2}\right\rfloor} a_{2 i}\left(G^{\sigma}\right) \lambda^{n-2 i}
$$

satisfies $a_{2 i}\left(G^{\sigma}\right) \geq 0$ for each $i\left(0 \leq i \leq\left\lfloor\frac{n}{2}\right\rfloor\right)$.

Proof. Let $\lambda_{1}, \lambda_{2}, \cdots, \lambda_{n}$ be all eigenvalues of the skew adjacency matrix $S\left(G^{\sigma}\right)$ of $G^{\sigma}$. Because $\lambda_{1}, \lambda_{2}, \cdots, \lambda_{n}$ are all purely imaginary numbers and must occur in 
conjugate pairs, we suppose, without loss of generality, that there exists an integer number $m\left(\leq\left\lfloor\frac{n}{2}\right\rfloor\right)$ such that

$$
\lambda_{t}=-\lambda_{n-t+1}=p_{t} i, \quad \text { for } t=1,2, \cdots, m,
$$

and all other eigenvalues are zero, where each $p_{t}$ is a positive real number and $i$ satisfies $i^{2}=-1$. Then we have

$$
\begin{aligned}
\phi\left(G^{\sigma}, \lambda\right) & =\prod_{t=1}^{n}\left(\lambda-\lambda_{t}\right) \\
& =\lambda^{n-2 m} \prod_{t=1}^{m}\left(\lambda^{2}+p_{t}^{2}\right),
\end{aligned}
$$

which implies that the result follows.

For an oriented graph $G^{\sigma}$ on $n$ vertices, an integral formula for the skew energy in terms of the skew characteristic polynomial $\phi\left(G^{\sigma}, \lambda\right)$ and its derivative is given by [1]

$$
\mathscr{E}_{s}\left(G^{\sigma}\right)=\frac{1}{\pi} \int_{-\infty}^{+\infty}\left[n+\lambda \frac{\phi^{\prime}\left(G^{\sigma},-\lambda\right)}{\phi\left(G^{\sigma},-\lambda\right)}\right] d \lambda .
$$

However, using the above integral, it is by no means easy to calculate the skew energy of an oriented graph. Hence, it is rather important to establish some other more simpler formula.

Applying to (2.1) the fact that the coefficient $a_{i}=0$ for each odd $i$ from Lemma 2 and replacing $\lambda$ by $-\lambda$, we have

$$
\mathscr{E}_{s}\left(G^{\sigma}\right)=\frac{1}{\pi} \int_{-\infty}^{+\infty}\left[n-\lambda \frac{\phi^{\prime}\left(G^{\sigma}, \lambda\right)}{\phi\left(G^{\sigma}, \lambda\right)}\right] d \lambda .
$$

Meanwhile, note that

$$
\frac{\phi^{\prime}\left(G^{\sigma}, \lambda\right)}{\phi\left(G^{\sigma}, \lambda\right)} d \lambda=d \ln \phi\left(G^{\sigma}, \lambda\right)
$$

Then we have

$$
\begin{aligned}
\mathscr{E}_{s}\left(G^{\sigma}\right) & =\frac{1}{\pi} \int_{-\infty}^{+\infty}\left[n-\lambda \frac{\phi^{\prime}\left(G^{\sigma}, \lambda\right)}{\phi\left(G^{\sigma}, \lambda\right)}\right] d \lambda \\
& =\frac{1}{\pi} \int_{-\infty}^{+\infty}\left[n-\lambda\left(\frac{d}{d \lambda}\right) \ln \phi\left(G^{\sigma}, \lambda\right)\right] d \lambda
\end{aligned}
$$

Therefore, we have

Theorem 5 Let $G^{\sigma}$ be an oriented graph with order $n$. Then

$$
\mathscr{E}_{s}\left(G^{\sigma}\right)=\frac{1}{\pi} \int_{-\infty}^{+\infty} \lambda^{-2} \ln \psi\left(G^{\sigma}, \lambda\right) d \lambda,
$$


where

$$
\psi\left(G^{\sigma}, \lambda\right)=\sum_{i=0}^{\left\lfloor\frac{n}{2}\right\rfloor} a_{2 i}\left(G^{\sigma}\right) \lambda^{2 i}
$$

and $a_{2 i}\left(G^{\sigma}\right)$ denotes the coefficient of $\lambda^{n-2 i}$ in the skew characteristic polynomial $\phi\left(G^{\sigma}, \lambda\right)$.

Proof. Let both $G_{1}^{\sigma_{1}}$ and $G_{2}^{\sigma_{2}}$ be oriented graphs with order $n$. ( $G_{1}$ perhaps equals $G_{2}$.) Then applying $(2.2)$ we have

$$
\mathscr{E}_{s}\left(G_{1}^{\sigma_{1}}\right)-\mathscr{E}_{s}\left(G_{2}^{\sigma_{2}}\right)=-\frac{1}{\pi} \int_{-\infty}^{+\infty} \lambda\left(\frac{d}{d \lambda}\right) \ln \left[\frac{\phi\left(G_{1}^{\sigma_{1}}, \lambda\right)}{\phi\left(G_{2}^{\sigma_{2}}, \lambda\right)}\right] d \lambda .
$$

Using partial integration, we have

$$
\mathscr{E}_{s}\left(G_{1}^{\sigma_{1}}\right)-\mathscr{E}_{s}\left(G_{2}^{\sigma_{2}}\right)=-\left.\frac{\lambda}{\pi} \ln \left[\frac{\phi\left(G_{1}^{\sigma_{1}}, \lambda\right)}{\phi\left(G_{2}^{\sigma_{2}}, \lambda\right)}\right]\right|_{-\infty} ^{+\infty}+\frac{1}{\pi} \int_{-\infty}^{+\infty} \ln \left[\frac{\phi\left(G_{1}^{\sigma_{1}}, \lambda\right)}{\phi\left(G_{2}^{\sigma_{2}}, \lambda\right)}\right] d \lambda .
$$

Notice that

$$
\left.\frac{\lambda}{\pi} \ln \left[\frac{\phi\left(G_{1}^{\sigma_{1}}, \lambda\right)}{\phi\left(G_{2}^{\sigma_{2}}, \lambda\right)}\right]\right|_{-\infty} ^{+\infty}=0
$$

Hence

$$
\mathscr{E}_{s}\left(G_{1}^{\sigma_{1}}\right)-\mathscr{E}_{s}\left(G_{2}^{\sigma_{2}}\right)=\frac{1}{\pi} \int_{-\infty}^{+\infty} \ln \left[\frac{\phi\left(G_{1}^{\sigma_{1}}, \lambda\right)}{\phi\left(G_{2}^{\sigma_{2}}, \lambda\right)}\right] d \lambda
$$

Suppose now that $G_{2}^{\sigma_{2}}$ is the null oriented graph, an oriented graph containing $n$ isolated vertices. Then $\phi\left(G_{2}^{\sigma_{2}}, \lambda\right)=\lambda^{n}$ and thus $\mathscr{E}_{s}\left(G_{2}^{\sigma_{2}}\right)=0$. After an appropriate change of variables we can derive

$$
\mathscr{E}_{s}\left(G_{1}^{\sigma_{1}}\right)=\frac{1}{\pi} \int_{-\infty}^{+\infty} \lambda^{-2} \ln \psi\left(G_{1}^{\sigma_{1}}, \lambda\right) d \lambda
$$

Then the result follows.

\section{Proof of Theorem 1}

From Theorem 5, for an oriented graph $G^{\sigma}$ on $n$ vertices, the skew energy $\mathcal{E}_{s}\left(G^{\sigma}\right)$ is a strictly monotonically increasing function of the coefficients $a_{2 k}\left(G^{\sigma}\right)\left(k=0,1, \cdots,\left\lfloor\frac{n}{2}\right\rfloor\right)$, since for each $i$ the coefficient of $\lambda^{n-i}$ in the characteristic polynomial $\phi\left(G^{\sigma}, \lambda\right)$, as well as $\psi\left(G^{\sigma}, \lambda\right)$, satisfies $a_{i}\left(G^{\sigma}\right) \geq 0$ by Lemma 4. Thus, similar to comparing two undirected graphs with respect to their energies, we define the quasi-ordering relation " $\preceq$ " of two oriented graphs with respect to their skew energies as follows. 
Let $G_{1}^{\sigma_{1}}$ and $G_{2}^{\sigma_{2}}$ be two oriented graphs of order $n$. $\left(G_{1}\right.$ is not necessarily different from $G_{2}$.) If $a_{2 i}\left(G_{1}^{\sigma_{1}}\right) \leq a_{2 i}\left(G_{2}^{\sigma_{2}}\right)$ for all $i$ with $0 \leq i \leq\left\lfloor\frac{n}{2}\right\rfloor$, then we write that $G_{1}^{\sigma_{1}} \preceq G_{2}^{\sigma_{2}}$.

Furthermore, if $G_{1}^{\sigma_{1}} \preceq G_{2}^{\sigma_{2}}$ and there exists at least one index $i$ such that $a_{2 i}\left(G_{1}^{\sigma_{1}}\right)<a_{2 i}\left(G_{2}^{\sigma_{2}}\right)$, then we write that $G_{1}^{\sigma_{1}} \prec G_{2}^{\sigma_{2}}$. If $a_{2 i}\left(G_{1}^{\sigma_{1}}\right)=a_{2 i}\left(G_{2}^{\sigma_{2}}\right)$ for all $i$, we write $G_{1}^{\sigma_{1}} \sim G_{2}^{\sigma_{2}}$. Note that there are non-isomorphic oriented graphs $G_{1}^{\sigma_{1}}$ and $G_{2}^{\sigma_{2}}$ such that $G_{1}^{\sigma_{1}} \sim G_{2}^{\sigma_{2}}$, which implies that " $\preceq$ " is not a partial ordering in general.

According to the integral formula (2.2), we have, for two oriented graphs $D_{1}$ and $D_{2}$ of order $n$, that

$$
D_{1} \preceq D_{2} \Longrightarrow \mathcal{E}_{s}\left(D_{1}\right) \leq \mathcal{E}_{s}\left(D_{2}\right)
$$

and

$$
D_{1} \prec D_{2} \Longrightarrow \mathcal{E}_{s}\left(D_{1}\right)<\mathcal{E}_{s}\left(D_{2}\right) .
$$

In the following, by discussing the relation " $\succeq$ ", we compare the skew energies for two oriented graphs and then complete the proof of Theorem 11.

Firstly, by a directly calculation we have

$$
\phi\left(O_{n, m}^{+}\right)=\lambda^{n}+m \lambda^{n-2}+(m-n+1)(2 n-m-3) \lambda^{n-4},
$$

and

$$
\phi\left(B_{n, m}^{+}\right)=\lambda^{n}+m \lambda^{n-2}+(m-n+2)(2 n-m-4) \lambda^{n-4} .
$$

Denote by $G^{\sigma}(n, m)$ and $G(n, m)$ the sets of all connected oriented graphs and undirected graphs with $n$ vertices and $m$ edges, respectively. The following results on undirected graphs are needed.

Lemma 6 Let $n \geq 5$ and $G \in G(n, m)$ be an arbitrary connected undirected graph containing $n$ vertices and $m(n \leq m<2(n-2))$ edges. Then $q(G) \leq\left(\begin{array}{c}m-n+2 \\ 2\end{array}\right)$, where $q(G)$ denotes the number of quadrangles contained in $G$.

Proof. We prove this result by induction on $m$.

The result is obvious for $m=n$. So we suppose that $n<m<2(n-2)$ and the result is true for smaller $m$.

Let $e$ be an edge of $G$ and $q_{G}(e)$ denote the number of quadrangles containing the edge $e$. Suppose $e=(u, v)$. Let $U$ be the set of neighbors of $u$ except $v$, and $V$ the set of neighbors of $v$ except $u$. Then there are just $q_{G}(e)$ edges between $U$ and 
$V$. Let $X$ be the subset of $U$ such that each vertex in $X$ is incident to some of the above $q_{G}(e)$ edges and $Y$ be the subset of $V$ defined similarly to $X$. Assume $|X|=x$ and $|Y|=y$. Let $G_{0}$ be the subgraph of $G$ induced by $V\left(G_{0}\right)=u \cup v \cup X \cup Y$. Then there are at least $q_{G}(e)+x+y+1$ edges and exactly $x+y+2$ vertices in $G_{0}$. In order for the remaining vertices to connect to $G_{0}$, the number of remaining edges must be not less than that of the remaining vertices. Thus

$$
m-\left(q_{G}(e)+x+y+1\right) \geq n-(x+y+2) .
$$

That is

$$
q_{G}(e) \leq m-n+1
$$

By induction hypothesis, $q(G-e) \leq\left(\begin{array}{c}(m-1)-n+2 \\ 2\end{array}\right)=\left(\begin{array}{c}m-n+1 \\ 2\end{array}\right)$. Then we have

$$
q(G)=q_{G}(e)+q(G-e) \leq m-n+1+\left(\begin{array}{c}
m-n+1 \\
2
\end{array}\right)=\left(\begin{array}{c}
m-n+2 \\
2
\end{array}\right) .
$$

Hence, the result follows.

By a similar method, we can show that

Lemma 7 Let $n \geq 5$ and $G \in G(n, m)$ be an arbitrary undirected graph containing $n$ vertices and $m(n \leq m<2(n-2))$ edges. Suppose $\Delta(G)=n-1$. Then

$$
q(G) \leq\left(\begin{array}{c}
m-n+1 \\
2
\end{array}\right)
$$

Lemma 8 [8, A part of Theorem 2.6] Let $G^{\sigma}$ be an oriented graph with an arc $e=(u, v)$. Suppose that $e$ is not contained in any even cycle. Then

$$
\phi\left(G^{\sigma}, \lambda\right)=\phi\left(G^{\sigma}-e, \lambda\right)+\phi\left(G^{\sigma}-u-v, \lambda\right) .
$$

As a consequence of Lemma 8, we have the following result.

Lemma 9 Let $G^{\sigma}$ be an oriented graph on $n$ vertices and $(u, v)$ a pendant arc of $G^{\sigma}$ with pendant vertex $v$. Suppose $\phi\left(G^{\sigma}, \lambda\right)=\sum_{i=0}^{n} a_{i}\left(G^{\sigma}\right) \lambda^{n-i}$. Then

$$
a_{i}\left(G^{\sigma}\right)=a_{i}\left(G^{\sigma}-v\right)+a_{i-2}\left(G^{\sigma}-v-u\right) .
$$

Based on the preliminary results above, we have the following two results. 
Lemma 10 Let $n \geq 5$ and $G^{\sigma} \in G^{\sigma}(n, m)$ be an oriented graph with maximum degree $n-1$. Suppose that $n \leq m<2(n-2)$ and $G^{\sigma} \nsim O_{n, m}^{+}$. Then $G^{\sigma} \succ O_{n, m}^{+}$.

Proof. By Theorem 3, it suffices to prove that $a_{4}\left(G^{\sigma}\right)>a_{4}\left(O_{n, m}^{+}\right)$. Suppose that $v$ is the vertex with degree $n-1$. For convenience, all arcs incident to $v$ are colored as white and all other arcs are colored as black. Then there are $n-1$ white arcs and $m-n+1$ black arcs. We estimate the cardinality of 2-matchings in $G^{\sigma}$ as follows. Noticing that all white arcs are incident to $v$, each pair of white arc can not form a 2-matching of $G^{\sigma}$. Since $d(v)=n-1$ and each black arc incident to exactly two white arcs, each black arc together with a white arcs except its neighbors forms a 2-matching of $G^{\sigma}$, that is, there are $(m-n+1)(n-3)$ black-white 2-matchings. Moreover, noticing that $G^{\sigma} \neq O_{n, m}^{+}, G^{\sigma}-v$ does not contain the directed star $S_{m-n+2}$ as its subgraph, and thus there is at least one 2-matching formed by a pair of disjoint black arcs, or $G^{\sigma}$ is an oriented graph of the following graph $F$.

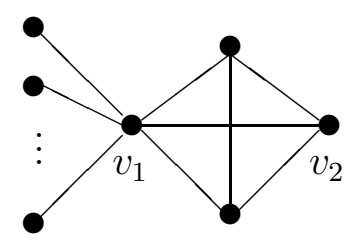

Fig. 1.2. The graph $F$.

If it is the first case, then the number of 2-matchings in $G^{\sigma}$ satisfies

$$
M\left(G^{\sigma}, 2\right) \geq(m-n+1)(n-3)+1 .
$$

From Lemma 7, $q\left(G^{\sigma}\right) \leq\left(\begin{array}{c}m-n+1 \\ 2\end{array}\right)$, and then by applying Theorem 3 again, we have

$$
\begin{aligned}
a_{4}\left(G^{\sigma}\right) & \geq M\left(G^{\sigma}, 2\right)-2 q\left(G^{\sigma}\right) \\
& \geq(m-n+1)(n-3)+1-2\left(\begin{array}{c}
m-n+1 \\
2
\end{array}\right) \\
& =a_{4}\left(O_{n, m}^{+}\right)+1
\end{aligned}
$$

by Eq.(3.2). If it is the second case, clearly $m=n+2, q(F)=3$, but the three quadrangles can not be all evenly oriented. Then

$$
a_{4}(F) \geq M(F, 2)-2 q(F) \geq(m-n+1)(n-3)-4>a_{4}\left(O_{n, n+2}^{+}\right) .
$$

The result thus follows. 
Lemma 11 Let $n \geq 5$ and $G^{\sigma} \in G^{\sigma}(n, m)$ be an oriented graph with $n \leq m<$ $2(n-2)$. Suppose that $\Delta\left(G^{\sigma}\right) \leq n-2$ and $G^{\sigma} \nsim B_{n, m}^{+}$. Then $G^{\sigma} \succ B_{n, m}^{+}$.

Proof. By Theorem 3 again, it suffices to prove that $a_{4}\left(G^{\sigma}\right)>a_{4}\left(B_{n, m}^{+}\right)$. We apply induction on $n$ to prove it. By a direct calculation, the result follows if $n=5$, since then $5=m<2(5-2)=6$ and there exists exactly four graphs in $G^{\sigma}(5,5)$, namely, the oriented cycle $C_{3}$ together with two pendant arcs attached to two different vertices of the $C_{3}$, the oddly oriented cycle $C_{4}$ together with a pendant arc, $B_{5,5}^{+}$and the oriented cycle $C_{5}$. Suppose now that $n \geq 6$ and the result is true for smaller $n$.

Case 1. There is a pendant arc $(u, v)$ in $G^{\sigma}$ with pendant vertex $v$.

By Lemma 9 we have

$$
a_{4}\left(G^{\sigma}\right)=a_{4}\left(G^{\sigma}-v\right)+a_{2}\left(G^{\sigma}-v-u\right)=a_{4}\left(G^{\sigma}-v\right)+e\left(G^{\sigma}-v-u\right) .
$$

Noticing that $\Delta\left(G^{\sigma}\right) \leq n-2$, we have $e\left(G^{\sigma}-v-u\right) \geq m-\Delta\left(G^{\sigma}\right) \geq m-n+2$.

By induction hypothesis, $a_{4}\left(G^{\sigma}-v\right) \geq a_{4}\left(B_{n-1, m-1}^{+}\right)$with equality if and only if $G^{\sigma}-v=B_{n-1, m-1}^{+}$. Then

$$
\begin{aligned}
a_{4}\left(G^{\sigma}\right) & =a_{4}\left(G^{\sigma}-v\right)+a_{2}\left(G^{\sigma}-v-u\right) \\
& \geq a_{4}\left(B_{n-1, m-1}^{+}\right)+m-n+2 \\
& =a_{4}\left(B_{n-1, m-1}^{+}\right)+e\left(S_{m-n+1}\right) \\
& =a_{4}\left(B_{n, m}\right)
\end{aligned}
$$

with equality if and only if $G^{\sigma}=B_{n, m}^{+}$. The result thus follows.

Case 2. There are no pendant vertices in $G^{\sigma}$.

Let

$$
(d)_{G^{\sigma}}=\left(d_{1}, d_{2}, \cdots, d_{i}, d_{i+1}, \cdots, d_{n}\right)
$$

be the non-increasing degree sequence of $G^{\sigma}$. We label the vertices of $G^{\sigma}$ corresponding to the degree sequence $(d)_{G^{\sigma}}$ as $v_{1}, v_{2}, \cdots, v_{n}$ such that $d_{G^{\sigma}}\left(v_{i}\right)=d_{i}$ for each $i$. Assume $d_{1}<n-2$. Then there exists a vertex $v_{k}$ that is not adjacent to $v_{1}$, but is adjacent to one neighbor, say $v_{i}$, of $v_{1}$. Thus

$$
\left(d_{1}+1, d_{2}, \cdots d_{i}-1, d_{i+1}, \cdots, d_{n}\right)
$$

is the degree sequence of the oriented graph $D^{\prime}$ obtained from $G^{\sigma}$ by deleting the $\operatorname{arc}\left(v_{k}, v_{i}\right)$ and adding the arc $\left(v_{k}, v_{1}\right)$, regardless the orientation of the $\operatorname{arc}\left(v_{k}, v_{1}\right)$. Rewriting the sequence above such that

$$
(d)_{D^{\prime}}=\left(d_{1}^{\prime}, d_{2}^{\prime}, \cdots, d_{i}^{\prime}, d_{i+1}^{\prime}, \cdots, d_{n}^{\prime}\right)
$$


is also a non-increasing sequence. Then $d_{1} \geq d_{i} \geq 2$ and thus we have

$$
\sum_{i=1}^{n}\left(\begin{array}{c}
d_{i}^{\prime} \\
2
\end{array}\right)>\sum_{i=1}^{n}\left(\begin{array}{c}
d_{i} \\
2
\end{array}\right)
$$

since

$$
\begin{aligned}
\sum_{i=1}^{n}\left(\begin{array}{c}
d_{i}^{\prime} \\
2
\end{array}\right)-\sum_{i=1}^{n}\left(\begin{array}{c}
d_{i} \\
2
\end{array}\right) & =\left(\begin{array}{c}
d_{1}+1 \\
2
\end{array}\right)+\left(\begin{array}{c}
d_{i}-1 \\
2
\end{array}\right)-\left(\begin{array}{c}
d_{1} \\
2
\end{array}\right)-\left(\begin{array}{c}
d_{i} \\
2
\end{array}\right) \\
& =d_{1}-d_{i}+1 \\
& >0 .
\end{aligned}
$$

Repeating this procedure, we can eventually obtain a non-increasing graph sequence

$$
(d)_{D^{\prime \prime}}=\left(d_{1}^{\prime \prime}, d_{2}^{\prime \prime}, \cdots, d_{i}^{\prime \prime}, d_{i+1}^{\prime \prime}, \cdots, d_{n}^{\prime \prime}\right)
$$

such that $\Delta\left(D^{\prime \prime}\right)=d_{1}^{\prime \prime}=n-2$ and

$$
\sum_{v \in D^{\prime \prime}}\left(\begin{array}{c}
d^{\prime \prime}(v) \\
2
\end{array}\right)>\sum_{v \in D^{\prime}}\left(\begin{array}{c}
d^{\prime}(v) \\
2
\end{array}\right)>\cdots>\sum_{v \in G^{\sigma}}\left(\begin{array}{c}
d(v) \\
2
\end{array}\right)
$$

Similarly, we can assume that there exists a vertex $v_{k}$ that is not adjacent to $v_{i}$, but is adjacent to one neighbor, say $v_{j}$, of $v_{i}$. Thus

$$
\left(d_{1}, d_{2}, \cdots d_{i}+1, d_{i+1}, \cdots, d_{j}-1, d_{j+1}, \cdots, d_{n}\right)
$$

is the degree sequence of the oriented graph $D^{\prime \prime \prime}$ obtained from $D^{\prime \prime}$ by deleting the arc $\left(v_{k}, v_{j}\right)$ and adding the arc $\left(v_{k}, v_{i}\right)$, regardless the orientation of the arc $\left(v_{k}, v_{j}\right)$. By a similar proof, we can get

$$
\sum_{v \in D^{\prime \prime \prime}}\left(\begin{array}{c}
d^{\prime \prime \prime}(v) \\
2
\end{array}\right)>\sum_{v \in D^{\prime \prime}}\left(\begin{array}{c}
d^{\prime \prime}(v) \\
2
\end{array}\right)
$$

Then by applying the above procedure repeatedly, we eventually obtain the degree sequence $(d)_{B_{n, m}^{+}}$,

$$
(d)_{B_{n, m}^{+}}=(n-2, m-n+2,2,2, \cdots, 2,1,1, \cdots, 1)
$$

where the number of vertices of degree 2 is $m-n+2$, and the number of vertices of degree 1 is $2 n-m-4$. Finally, we get

$$
\sum_{v \in B_{n, m}^{+}}\left(\begin{array}{c}
d^{B^{+}}(v) \\
2
\end{array}\right)>\sum_{v \in D^{\prime \prime \prime}}\left(\begin{array}{c}
d^{\prime \prime \prime}(v) \\
2
\end{array}\right)>\sum_{v \in D^{\prime \prime}}\left(\begin{array}{c}
d^{\prime \prime}(v) \\
2
\end{array}\right)>\cdots>\sum_{v \in G^{\sigma}}\left(\begin{array}{c}
d(v) \\
2
\end{array}\right) .
$$


Then the lemma follows by combining Eq.(3.3) and Lemma 6 with the fact that $M(G, 2)=\left(\begin{array}{c}m \\ 2\end{array}\right)-\sum_{v \in G^{\sigma}}\left(\begin{array}{c}d(v) \\ 2\end{array}\right)$.

Combining Lemma 10 with Lemma 11, we get the proof of Theorem 11 immediately.

Proof of Theorem 1. Combining with Lemmas 10 and 11, the oriented graph with minimal skew energy among all oriented graphs of $G^{\sigma}(n, m)$ with $n \leq m \leq 2(n-2)$ is either $O_{n, m}^{+}$or $B_{n, m}^{+}$. Furthermore, from (3.2) and (3.3), we have

$$
a_{4}\left(O_{n, m}^{+}\right)=(m-n+1)(2 n-m-3)
$$

and

$$
a_{4}\left(B_{n, m}^{+}\right)=(m-n+2)(2 n-m-4) .
$$

Then, by a direct calculation we have $a_{4}\left(O_{n, m}^{+}\right)<a_{4}\left(B_{n, m}^{+}\right)$if $m<\frac{3 n-5}{2} ; a_{4}\left(B_{n, m}^{+}\right)=$ $a_{4}\left(O_{n, m}^{+}\right)$if $m=\frac{3 n-5}{2}$; and $a_{4}\left(O_{n, m}^{+}\right)>a_{4}\left(B_{n, m}^{+}\right)$otherwise. The proof is thus complete by $(3.1)$.

\section{References}

[1] C. Adiga, R. Balakrishnan and Wasin So, The skew energy of a graph, Linear Algebra Appl. 432 (2010), 1825-1835.

[2] F. Alinaghipour and B. Ahmadi, On the energy of complement of regular line graph, MATCH Commun. Math. Comput. Chem. 60(2008), 427-434.

[3] S. Akbari, E. Ghorbani and M.R. Oboudi, Edge addition, singular values and energy of graphs and matrices, Linear Algebra Appl. 430(2009): 2192-2199.

[4] S.R. Blackburn and I.E. Shparlinski, On the average energy of circulant graphs, Linear Algebra Appl. 428 (2008), 1956-1963.

[5] G. Caporossi, D. Cvetković, I. Gutman and P. Hansen, Variable neighborhood search for extremal graphs. 2. Finding graphs with external energy, J. Chem. Inf. Comput. Sci. 39 (1999), 984-996.

[6] C.A. Coulson, On the calculation of the energy in unsaturated hydrocarbon molecules, Proc. Cambridge Phil. Soc. 36(1940), 201-203.

[7] J. Day and W. So, Graph energy change due to edge deletion, Linear Algebra Appl., 428 (2007): 2070-2078. 
[8] S. Gong and G. Xu, The characteristic polynomial and the matchings polynomial of a weighted oriented graph, Linear Algebra Appl. 436(2012), 3597-3607.

[9] S. Gong, G. Xu, 3-Regular digraphs with optimum skew energy, Linear Algbra Appl. 436(2012), 465-471.

[10] I. Gutman, The energy of a graph, Ber. Math.-Statist. Sekt. Forschungsz. Graz. 103(1978), 1-22.

[11] I. Gutman, The energy of a graph: old and new results, in: A. Betten, A. Kohnert, R. Laue and A. Wassermann(Eds.), Algebraic Combinatorics and Applications, Springer, Berlin, 2000, pp.196-211.

[12] I. Gutman, D. Kiani and M. Mirzakhah, On incidence energy of graphs, MATCH Commun. Math. Comput. Chem. 62 (2009), 573-580.

[13] I. Gutman, D. Kiani, M. Mirzakhah and B. Zhou, On incidence energy of a graph, Linear Algebra Appl. 431 (2009), 1223-1233.

[14] I. Gutman and O.E. Polansky, Mathematical Concepts in Organic Chemistry, Springer, Berlin, 1986.

[15] I. Gutman, M. Robbiano, E.A. Martins, D.M. Cardoso, L. Medina and O. Rojo, Energy of line graphs, Linear Algebra Appl. 433(2010), 1312-1323.

[16] I. Gutman and B. Zhou, Laplacian energy of a graph, Linear Algebra Appl. 414(2006), 29-37.

[17] Y. Hou, Unicyclic graphs with minimal energy, J. Math. Chem. 29(2001), 163168.

[18] Y. Hou, Bicyclic graphs with minimum energy, Linear and Multilinear Algebra 49(2001), 347-354.

[19] Y. Hou and T. Lei, Characteristic polynomials of skew-adjacency matrices of oriented graphs, Electron. J. Combin. 18(2011), \#p156.

[20] Y. Hou, X. Shen and C. Zhang, Oriented unicyclic graphs with extremal skew energy, Avialable at http://arXiv.org/abs/1108.6229.

[21] X. Li, Y. Shi and I. Gutman, Graph Energy, Springer, New York, 2012.

[22] X. Li, J. Zhang and L. Wang, On bipartite graphs with minimal energy, Discrete Appl. Math. 157(2009), 869-873. 
[23] X. Shen, Y. Hou and C. Zhang, Bicyclic digraphs with extremal skew energy, Electron. J. Linear Algbra 23(2012), 340-355.

[24] G. Tian, On the skew energy of orientations of hypercubes, Linear Algbra Appl. 435(2011), 2140-2149.

[25] J. Zhang and B. Zhou, On bicyclic graphs with minimal energies, J. Math. Chem. 37(4)(2005), 423-431.

[26] J. Zhu, Oriented unicyclic graphs with the first $\left\lfloor\frac{n-9}{2}\right\rfloor$ largest skew energies, Linear Algebra Appl. 437(2012), 2630-2649. 